\title{
Clinical findings and outcomes in patients with pyoderma gangrenosum: A single tertiary centre experience
}

\author{
Pinar Incel Uysal, Gunes Gur Aksoy, Basak Yalcin
}

Department of Dermatology, Ankara Numune Training and Research Hospital, Ankara, Turkey

Corresponding author: Pinar Incel Uysal, MD, E-mail: pinarincel@hotmail.com

\begin{abstract}
Background: Pyoderma gangrenosum (PG) is an uncommon neutrophilic dermatosis which has a great variety of clinical presentation and course. Material and methods: We reviewed the medical records of PG patients who were diagnosed and treated in our department between 2015-2018 years. Results: Our study included 16 patients ( 10 female, 10 male). Lower extremity was the most common location (81\%). Of the 16 patients, 8 had multiple ulcerations at the time of diagnosis. Regarding associated comorbidities, haematological disorders (25\%) and seronegative arthritis (25\%) were the most frequent. Surgery and trauma were detected in two patients as a triggering factor. Pathergy positivity was documented in 6 patients. First-line immunosuppressive monotherapy was effective in 7 (43\%) patients. Mean duration to complete remission was 4.6 months. Conclusions: In our study we present our experience with some unusual aspects. Our series represented male predominance and higher rates of associated haematological disorders. Initial treatment with systemic corticosteroids is likely to be associated with faster response.
\end{abstract}

Key words: Cutaneous ulcers; Neutrophilic disorders; Pyoderma gangrenosum

\section{INTRODUCTION}

Pyoderma gangrenosum (PG) is an uncommon chronic inflammatory disorder with neutrophilic inflammation. PG is still a challenging disease which is poorly characterized in terms of diagnostic criteria and treatment outcome. Patients with PG usually present with one or more very painful ulcerations with violaceous undermined borders. Major clinical types of PG are as follows: ulcerative, pustular, bullous, and vegetating/ superficial granulomatous [1]. However, wide variety of clinical presentations and course can be seen. Exact pathogenetic mechanisms are not fully elucidated yet but association with other immune-mediated disorders and typically favorable treatment response to immunomodulatory drugs including corticosteroids, intravenous immunoglobulin, antitumor necrosis factor- $\alpha$ modalities, plasmapheresis support the immune mediated pathogenic pathways for PG [2].
PG is associated with underlying systemic comorbidites in $50-75 \%$ of the cases. Inflammatory bowel diseases, seronegative arthritis, heamatological disorders including malignancies and monoclonal gammopathy of undetermined significance (MGUS) and Behcet disease are common diseases related with PG. It has been reported that in pediatric population the association with inflammatory bowel disease is common[3] Also, surgical procedures may trigger the condition [4]. The disorder may arise as a component of syndromic forms which belong to a spectrum of autoinflammatory conditions characterized with over activated innate immune system [5]. These syndromes are PAPA (pyogenic arthritis, PG and acne), PASH (PG, acne and suppurative hidradenitis), PAPASH (pyogenic arthritis, acne, PG and suppurative hidradenitis) and PASS (pyoderma gangrenosum, acne conglobata, suppurative hidradenitis and seronegative spondiloarthropaty).

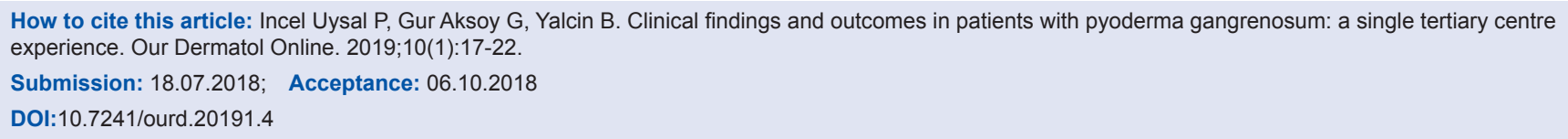


Since PG is considered an orphan disease, prospective randomized studies are lacking in the literature. In addition, due to the low incidence, there is still currently no uniform therapeutic approach. However, PG is potentially life threatening disorder which may show extracutanoeus manifestations [6]. Presence of systemic manifestations including renal and pulmonary involvement and association with comorbidities, seriously affect the choice of treatment.

The main aim of our study was to describe the characteristics of patients with PG in terms of demographics, clinical characteristics, comorbidities, coincidental findings, treatment modalities, and treatment outcomes. Here we document our observations on remarkable clinical findings of PG patients in order to help better understanding of this peculiar disorder.

\section{MATERIALS AND METHODS}

A retrospective single-center study was performed to evaluate the patients who were diagnosed with PG in our dermatology department between July 2015 and July 2018. In our department we reviewed the data of patients with the ICD code (L88) on medical charts. History and demographical data of the patients, clinical characteristics, laboratory analyses, associated diseases, coincidental findings, therapeutic interventions and recurrence during follow-up period were recorded. The patients with inadequate data, inconsisted diagnosis of PG or miscoded diagnosis were excluded. Subjects without histopathologic evidence of PG were not included. Follow-up data of the patients were obtained from both inpatient and outpatient medical records. Complete remission was defined as $100 \%$ healing of ulcer size or improvement all the lesions, partial remission as a decrease from $50 \%$ to $100 \%$ ulcer size or lesions. Patients with a $<50 \%$ decrease of ulcer size or improvement $<50 \%$ of lesions are grouped as persistent disease.

\section{Statistics}

Statistical analyses were carried out using SPSS software (version 21.0 for Windows; SPSS Inc, Chicago, IL, USA). Parametric variables were presented as means and standart deviations and nonparametric variables were presented as medians and interquartile ranges. For categorical variables number of cases and percentages were used. Kolmogorov Smirnov and histogram analyses were used to determine whether continous variables were normally distributed.

\section{Ethics}

The methods were in accordance with ethical principles of Declaration of Helsinki and the approval letter of ethics committee was obtained.

\section{RESULTS}

\section{Demographics and Clinical Characteristics}

Demographical and clinical data of the PG patients were summarized in the Table 1.

Among 34 patients with the presumptive diagnosis of PG during this 3-year period, 16 patients were finally diagnosed with PG. All of the PG patients included in this study required hospitalization. There were $10(62 \%)$ men and $6(38 \%)$ were women. The mean (SD) age at diagnosis was $41.5(12.3,25-59)$ years. The mean duration of disease was 3.5 months and median follow up time was 13 (range 6-22) months.

Twelve $(75 \%)$ of the recorded cases presented with classical(ulcerative) type. In 7 (43\%) of the patients, these ulcerative lesions were exclusively located on the lower limbs (Figs. la and lb). Fifty percent of the patients exhibited multiple ulcerations (Table 1). Cribriform scarring or wrinkled paper scars were observed at healed ulcer sites in $6(50 \%)$ patients with ulcerative lesions (Fig. 2).

Pathergy positivity was noted in $37 \%$ of the cases. Trauma was precipitating factor in two patients. One of the male patients reported that he had received wet cupping therapy for chronic leg swelling and pain two weeks before the occurrence of PG lesions (Fig. 3). Pustular type was observed in two patients and the bullous in two. There was no vegetative variant in our series. Three (18\%) patients had multiple attacks. Lower limbs were the most commonly affected site, mainly the pretibial areas.

\section{Comorbidities and Coincidental Findings}

In our series, $5(31 \%)$ of the patients had none of the comorbid diseases previously described in the literature (Table 1). Among these patients two patients had triggering factor: one with breast surgery and one with cupping treatment. Common comorbid diseases diagnosed before the occurrence of PG were listed in the Table 2. Type 2 diabetes mellitus(2), Hashimoto thyroiditis(1), deep venous thrombosis(1) were other medical conditions diagnosed before PG. 
Table 1: Data of the patients diagnosed with PG in our department between July 2015 and July 2018

\begin{tabular}{|c|c|c|c|}
\hline & $\operatorname{Men}(n=10)$ & Women $(n=6)$ & Total $(n=16)$ \\
\hline Age at onset, mean $\pm \mathrm{SD}$ & $37.4 \pm 11.1$ & $48.5 \pm 11.7$ & $41.5 \pm 12.3$ \\
\hline Duration of the disease, mean $\pm S D$, months & $4.7 \pm 7.1$ & $1.6 \pm 1.3$ & $3.5 \pm 5.8$ \\
\hline \multicolumn{4}{|l|}{ Initial presentation } \\
\hline Ulcer, n(\%) & $8(50 \%)$ & $4(25 \%)$ & $12(75 \%)$ \\
\hline Pustule, n(\%) & $1(6 \%)$ & $1(6 \%)$ & $2(12 \%)$ \\
\hline Blister, $n(\%)$ & $1(6 \%)$ & $1(6 \%)$ & $2(12 \%)$ \\
\hline Total number of ulcers & $2.6 \pm 1.6$ & $2.6 \pm 1.6$ & $2.6 \pm 1.6$ \\
\hline - Solitary ulcer, n (\%) & $2(12 \%)$ & $2(12 \%)$ & $4(25 \%)$ \\
\hline - Multiple ulcers( $\geq 2), n(\%)$ & $6(37 \%)$ & $2(12 \%)$ & $8(50 \%)$ \\
\hline Ulcer size, min-max, $\mathrm{cm}$ & $3-40$ & $2-8$ & $2-40$ \\
\hline Attack number, min-max & $1-4$ & 1 & $1-4$ \\
\hline Pathergy positivity, $\mathrm{n}(\%)$ & $4(25 \%)$ & $2(12 \%)$ & $6(37 \%)$ \\
\hline \multicolumn{4}{|l|}{ Localization } \\
\hline Lower extremity, $\mathrm{n}(\%)$ & $9(56 \%)$ & $4(25 \%)$ & $13(81 \%)$ \\
\hline Upper extremity, $\mathrm{n}(\%)$ & $3(18 \%)$ & - & $3(18 \%)$ \\
\hline Breast , $\mathrm{n}(\%)$ & - & $3(18 \%) 2(12 \%)$ & $3(18 \%)$ \\
\hline Trunk, $\mathrm{n}(\%)$ & - & & $2(12 \%)$ \\
\hline \multicolumn{4}{|l|}{ Triggering factors } \\
\hline Trauma (surgery), $\mathrm{n}(\%)$ & $1(6 \%)$ & $1(6 \%)$ & $2(12 \%)$ \\
\hline \multicolumn{4}{|l|}{ Comorbidities } \\
\hline - No comorbidites* & $3(18 \%)$ & $2(12 \%)$ & $5(31 \%)$ \\
\hline - One comorbidity & $4(25 \%)$ & $2(12 \%)$ & $6(37 \%)$ \\
\hline - Multiple comorbidity ( $\geq 2$ ) & $3(18 \%)$ & $2(12 \%)$ & $5(31 \%)$ \\
\hline
\end{tabular}

${ }^{*}$ Diabetes, autoimmune thyroid disorders and peripheral vascular disorders were not included.
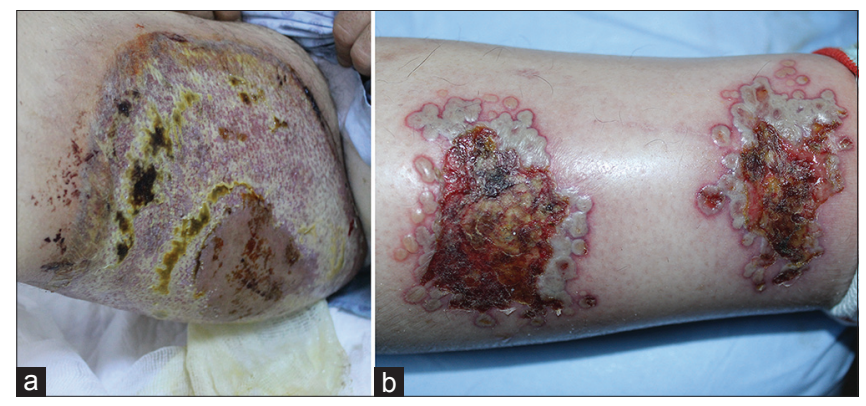

Figure 1: Initial presentation of a classical type PG in a 55-year old male patient without known triggering factor (1a). Multiple confluent vesicles and tiny vesico-pustules surrounding ulcerations in a female patient with coexistent multiple myeloma (1b).

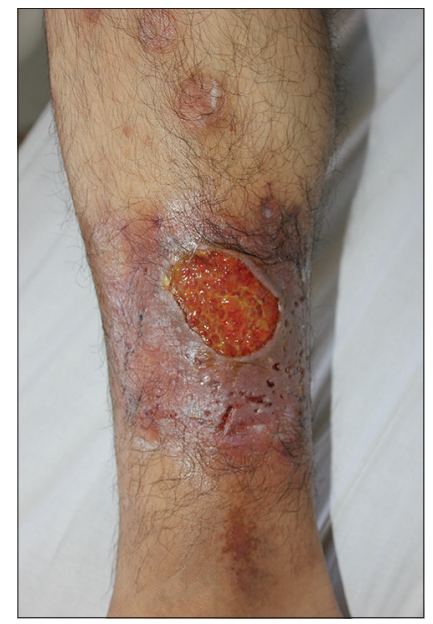

Figure 2: Characteristic appearance of healing period of an ulcerative PG demonstrating wrinkled-paper and cribriform scarring.

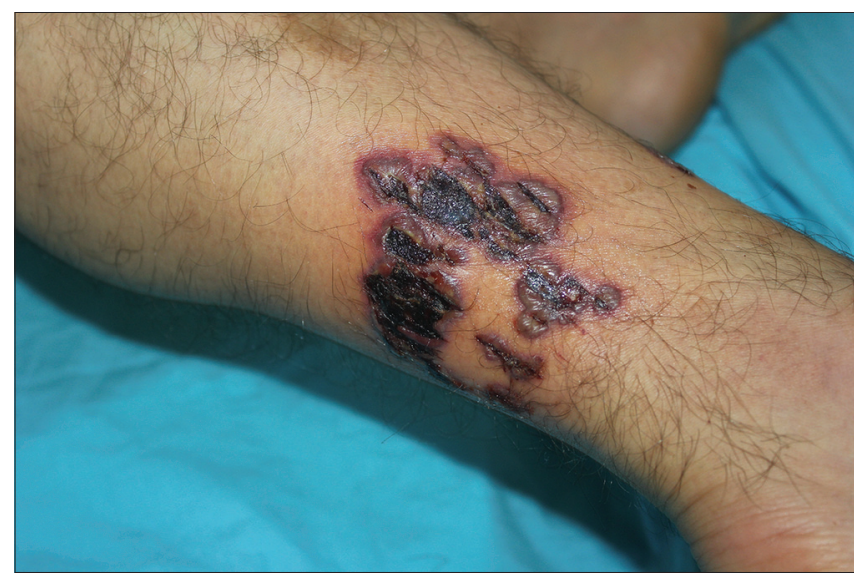

Figure 3: Hijama-induced multiple linear ulcerations with erythematous borders.

Extended laboratory investigations in our center revealed other concurrent diseases: sigmoid colonic polyp(1), monoclonal gammopathy of undetermined significance (MGUS)(1), cryoglobulinemia(1), hydatid cyst(1), multiple myeloma(1), factor V leiden mutation(1), benign thyroid nodules(2). One of the female patients with disseminated sterile pustules and tender nodules, developed multiple myeloma from preceding MGUS during follow up period (Fig. 4). Notably, one of the patients was under therapy for alcohol and cocaine addiction. Two of the patients who were suffered from ulcerative lesions with vegetating aspects were diagnosed with PASH (Fig. 5) and PASS 
Table 2: Comorbidites, coincidental findings and associated syndromes in the patients with PG.

\begin{tabular}{lc}
\hline Comorbidities & $\mathbf{n}(\%)$ \\
\hline Ulcerative colitis & $2(12)$ \\
Behcet disease & $2(12)$ \\
MGUS & $2(12)$ \\
Multiple myeloma ${ }^{\infty}$ & $3(18)$ \\
Psoriasis $_{\text {Psoriatic arthritis }}$ & $2(12)$ \\
Seronegative arthritis & $2(12)$ \\
\hline Coincidental conditions & $2(12)$ \\
\hline Sigmoid colonic polyp & $\mathbf{n}(\%)$ \\
Cryoglobulinemia, & $1(6)$ \\
Hydatid cyst & $1(6)$ \\
Factor V leiden mutation & $1(6)$ \\
Benign thyroid nodules & $1(6)$ \\
Peripheral vascular disease & $2(12)$ \\
Type 2 diabetes mellitus & $1(6)$ \\
Hashimoto thyroiditis & $2(12)$ \\
Syndromic forms & $1(6)$ \\
\hline$\infty$ One of the cases with MGUS progressed into MM during the follow-up & $2(12)$ \\
period. & \\
*Two male patients were diagnosed with PASS and PASH syndrome.
\end{tabular}

Table 3: Treatment regimes and outcomes in patients with PG.

\begin{tabular}{lc}
\hline Treatment modality & $\mathbf{n}(\%)$ \\
Immunosuppressive monotherapy & $10(10)$ \\
Immunosuppresive polytherapy & $4(25)$ \\
Exclusively topical wound care $\alpha$ & $2(12)$ \\
Systemic therapy & \\
Methylprednisolone* & $4(25)$ \\
Prednisolone* & $4(25)$ \\
Dapsone & $1(6)$ \\
Methotrexate & $1(6)$ \\
Pulse methylprednisolone $\alpha+$ Methotrexate (SC) & $1(6)$ \\
Methylprednisolone+Cyclosporine & $1(6)$ \\
Methylprednisolone+Mycophenolate mofetil & $1(6)$ \\
Prednisolone+Infiliximab & $1(6)$ \\
Infliximab & $1(6)$ \\
Tretment outcome & \\
Complete remission & $11(75)$ \\
Partial remission & $3(18)$ \\
Persistent disease & $1(6)$ \\
Contact lost to patient & $1(6)$ \\
Mean duration to complete remission, mean $\pm S D$, & $4.6 \pm 2.3$ \\
months & \\
Recurrence & $3(18)$ \\
\hline
\end{tabular}

*Glucocorticosteroid at 1-2 mg/kg/day with gradually tapering was used.

${ }^{\alpha} 1 \mathrm{~g} /$ day 5 consecutive days

syndrome because of the presence other clinical manifestations.

\section{Treatment Modalities}

Most patients (87.5\%) were treated with systemic therapy. Systemic corticosteroids were initial treatment regimen in 11 patients (68.7\%) either alone in 7 patients or in combination with cyclosporine in one $(6 \%)$, methotrexate in one $(6 \%)$, mycophenolate mofetil in one $(6 \%)$, infliximab in one $(6 \%)$. Corticosteroid and infliximab combination was chosen as first line therapy

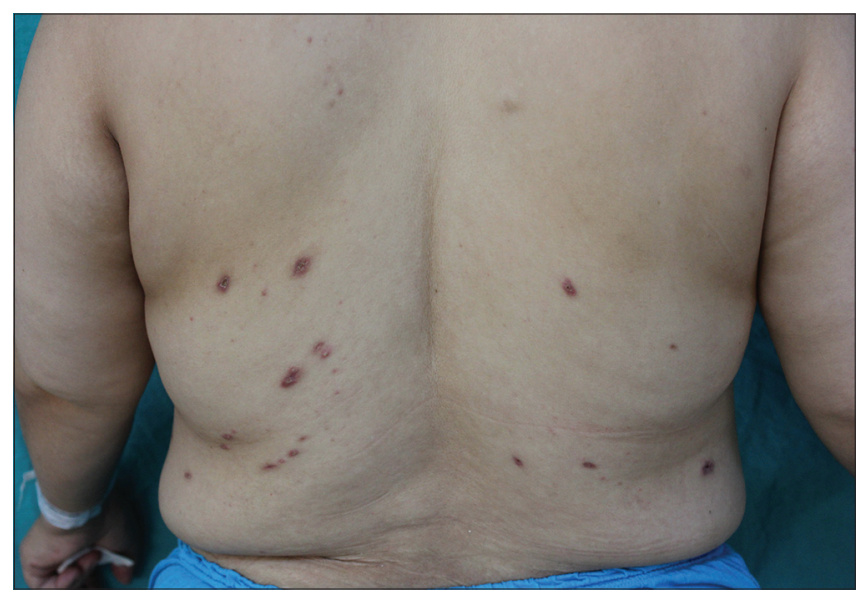

Figure 4: 44-year old female presenting with disseminated sterile pustules on the trunk in association with MGUS.

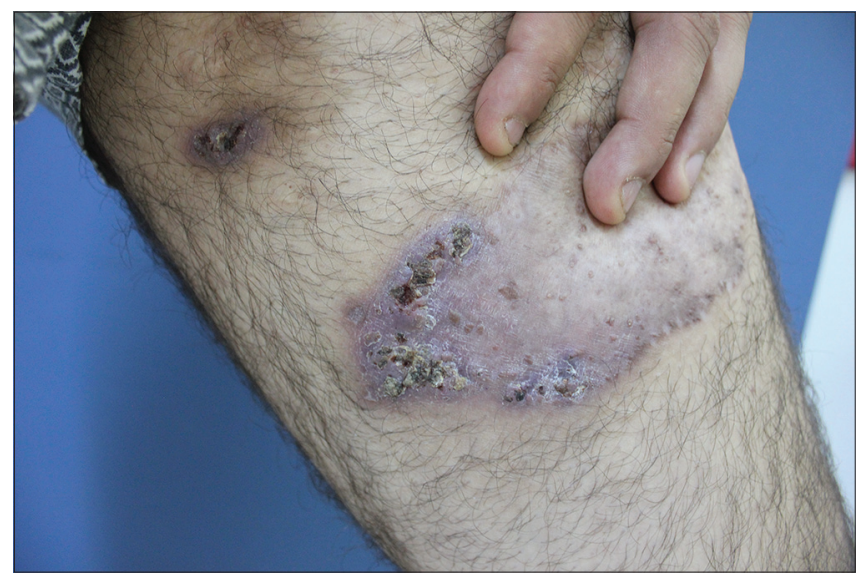

Figure 5: Vegetating appearance of an ulcerative lesion in a 28-year old male diagnosed with PASH syndrome.

for the patient with PASS syndrome. In three patients who had comorbidities that contraindicated the use of systemic steroids treatment regimens were as follows: dapsone(1), methotrexate(1) and cyclosporine(1). Topical wound care was carried out in all patients. Two patients treated with topical wound care and topical super-potent corticosteroids. Treatment regimens were included in Table 3.

\section{Treatment Outcomes}

Systemic therapy at first line was effective with complete healing in $9(56 \%)$ patients on average $4.4 \pm 1.3$ months. Patients (12\%) who were exclusively treated with topical wound care and topical corticosteroids eventually achieved complete response. One of three patients with partial response infliximab was added to initial therapy because of steroid failure. In a patient with ulcerative colitis who was free from PG for 10 months recurrence was noted after one year. Systemic antibiotic treatment was promptly initiated in five patients who had clinical 
findings such as erythema, swelling, fever and elevated C-reactive protein and positive culture from smears.

\section{DISCUSSION}

PG may affect any age group but those between $20-50$ years are particularly at risk $[1,7]$. Similarly, in our study mean age of onset of total study population was 41.5. Female patients were older than male patients at onset of disease however the difference did not reach statistical significance (Table 1). In contrast to many previous studies, there was men predominance (1.6:1) in our study group as it was reported by some authors [8].

Presentation with ulcerative lesions was the most common clinical type in our study as in other series [7,9]. Almost $81 \%$ of the PG lesions were located on lower extremity. Interestingly, one of these cases was triggered with wet cupping (or called 'hijama') therapy which is a form of alternative medicine (Fig. 3). In this technique before the cups are applied, tiny incisions are made in the skin for the elimination of toxic blood or fluids. We emphasize this patient because of distinct clinical picture with sterile bullae rapidly progressing to shallow ulceration. This patient with preceding hijama history, seem to be the first reported in the literature. He was successfully treated with corticosteroid monotherapy.

PG should be included in the differential diagnosis of breast ulcers. Among female patients (6) breast involvement was also common $(50 \%)$ in our series. It is well known that postoperative pyoderma gangrenosum commonly affects breasts [10]. One of our patients with bilaterally breast involvement had had breast reduction immediately before the occurrence of PG lesion. Recently, Maverakis et al. reported the results of Delphi consensus on diagnostic criteria of ulcerative PG [11]. In their report presence of multiple ulcers at least one anterior lower leg was one of the newly introduced diagnostic criteria. As they have stated we observed involvement of the anterior leg involvement in $58 \%$ of patients. Also, as the panel has agreed that cribriform or wrinkled paper scarring is useful in the diagnosis of PG, we observed these scars during healing process in half of the patients with ulcerations.

In the literature, percentage of the association with underlying diseases varies between 50\%-70\% [7, 2]. PG is associated with systemic diseases in $68.7 \%$ of our patients. It has been suggested that comorbidities including diabetes and PVD may be contributing factors for the development of PG [7]. However we did not include newly reported associations including endocrinopathies [12]. Hematological disorders were the most common underlying diseases in our series. Of note coincidental findings which were detected after the diagnosis of PG were considerable (Table 2). Interestingly two of our patients had the diagnosis of PG associated genetic syndromes (PASS and PASH syndrome) in our department. Taken together these results may suggest that individuals in a tertiary care setting are more likely to have concurrent serious or complicated diseases.

Several mucocutaneous diseases have been associated with cocaine consumption. Cocaine induced pyoderma gangrenosum (CIPG) is one of the cutaneous manifestations associated with cocaine use[13]. Clinically, lesions are not indistinguishable from other forms. However they tend to be of greater size and multiple [13]. A male patient with a history of cocaine abuse presented with multiple ulcerative lesions which were located on the upper extremities. He was treated with pulse methylprednisolone and methotrexate (15 mg/weekly, sc). All lesions healed within 4 months but recurrence was noted during follow-up. This would support that CIPG is seem to be more refractory and recurrent.

To date, there are still no established gold-standard treatment strategy and uniformly effective therapy for PG. The main aim is to promote wound healing and to reduce the pain by reducing inflammation. Milder forms of disease may be treated with local wound care [14]. In our clinical practice we usually prefer topical modalities in combination with systemic treatments. In this series, we had two patients who were exclusively controlled with topical wound therapy. Because of small sample size our study can provide little guidance regarding the choice of systemic therapy. However we observed shorter periods of healing in patients who were initially treated with systemic corticosteroids (usually oral corticosteroids). In our series, four patients required immunosuppresive polytherapy. All these patients had severe form of PG and severe comorbidities requiring faster taper of corticosteroids. Anti-tumor necrosis factor alfa (anti-TNF- $\alpha$ ) agents are another treatment option for resistant PG [15]. TNF- $\alpha$ is the main inflammatory mediator of PASS syndrome [5]. We preferred to use infliximab in two patients who had the diagnosis of Behcet disease and PASS syndromes. 
In our retrospective study of PG patients healing was achieved approximately within 4 months in $75 \%$ of the cases. Forty three percent of the patients were successfully treated with first-line immunosuppressive monotherapy. Our results were comparable with previous reports $[9,16]$. It has been reported that recurrence rates of PG is $17-61 \%$ [9]. In our series of 16 patients, only $3(18.7 \%)$ patients who were suffering from co-morbid (psoriatic arthritis in one, ulcerative colitis in one and seronegative spondyloarthropathy in one) chronic diseases had recurrent attacks.

Our study has several limitations related to its retrospective design and evaluation of small number of patients. So we were unable to detect a statistical difference in many of parameters between subgroups. Because of rarity the disorder it is quite difficult to present large number of cases. Nevertheless it is not possible for us to draw strong conclusions based on the data reported here.

\section{CONCLUSION}

These results described here represent our tertiary centre experience to date. Our study demonstrated a series of 16 patients with PG. Underlying systemic diseases were observed in $68 \%$ of the patients. Haematological disorders (MGUS and multiple myeloma) and seronegative arthritis were the most common (25\%) associated diseases. Development of new lesions after trauma and surgery was documented in two of the patients. Most of the patients (43\%) were controlled with first line immunosuppressive monotherapy, mainly systemic corticosteroid therapy. Mean duration to complete remission was 4.6 months. Recurrence was noted in three (18\%) individuals.

\section{Statement of Human and Animal Rights}

All procedures followed were in accordance with the ethical standards of the responsible committee on human experimentation (institutional and national) and with the Helsinki Declaration of 1975, as revised in 2008.

\section{REFERENCES}

1. Binus AM, Qureshi AA, Li VW, Winterfield LS. Pyoderma gangrenosum: a retrospective review of patient characteristics, comorbidities and therapy in 103 patients. Br J Dermatol. 2011;165:1244-50.

2. Kridin K, Cohen AD, Amber KT. Underlying Systemic Diseases in Pyoderma Gangrenosum: A Systematic Review and Meta-Analysis. Am J Clin Dermatol. 2018;19:479-87.

3. Diatta BA TF, Diop A, Diadie S, Ndiaye M, Diallo M, Niang SO, et al. Pyoderma gangrenosum among children in Senegal: 6 cases. Our Dermatol Online. 2017;8:463-6.

4. Hiraiwa T FH, Yamamoto T. Pyoderma gangrenosum triggered by surgical procedures in patients with underlying systemic diseases. Our Dermatol Online. 2014;5:432-3.

5. Cugno M, Borghi A, Marzano AV. PAPA, PASH and PAPASH Syndromes: Pathophysiology, Presentation and Treatment. Am J Clin Dermatol. 2017;18:555-62.

6. Wollina U. Pyoderma gangrenosum--a systemic disease? Clin Dermatol. 2015;33:527-30.

7. Adisen E, Erduran F, Gurer MA. Pyoderma Gangrenosum: A Report of 27 Patients. Int J Low Extrem Wounds. 2016;15:148-54.

8. Bhat RM, Nandakishore B, Sequeira FF, Sukumar D, Kamath GH, Martis J, et al. Pyoderma gangrenosum: an Indian perspective. Clin Exp Dermatol. 2011;36:242-7.

9. Pereira N, Brites MM, Goncalo M, Tellechea O, Figueiredo A. Pyoderma gangrenosum--a review of 24 cases observed over 10 years. Int J Dermatol. 2013;52:938-45.

10. Tolkachjov SN, Fahy AS, Cerci FB, Wetter DA, Cha SS, Camilleri MJ. Postoperative Pyoderma Gangrenosum: A Clinical Review of Published Cases. Mayo Clin Proc. 2016;91:1267-79.

11. Maverakis E, Ma C, Shinkai K, Fiorentino D, Callen JP, Wollina U, et al. Diagnostic Criteria of Ulcerative Pyoderma Gangrenosum: A Delphi Consensus of International Experts. JAMA Dermatol. 2018;154:461-6.

12. Abdel-Mohsen MA, El-Braky AA, Ghazal AAE, Shamseya MM. Autophagy, apoptosis, vitamin $\mathrm{D}$, and vitamin $\mathrm{D}$ receptor in hepatocellular carcinoma associated with hepatitis $C$ virus. Medicine (Baltimore). 2018;97:e0172.

13. Moreno-Artero E, Querol-Cisneros E, Rodriguez-Garijo N, TomasVelazquez A, Antonanzas J, Secundino F, et al. Mucocutaneous manifestations of cocaine abuse: a review. J Eur Acad Dermatol Venereol. 2018;32:1420-6.

14. Feldman SR, Lacy FA, Huang WW. The safety of treatments used in pyoderma gangrenosum. Expert Opin Drug Saf. 2018;17:55-61.

15. Ahronowitz I, Harp J, Shinkai K. Etiology and management of pyoderma gangrenosum: a comprehensive review. Am J Clin Dermatol. 2012;13:191-211.

16. Duman N, Evans SE, Karaduman A, Elçin G, Özaygen GE, Atakan N. et al. Pyoderma Gangrenosum: A Retrospective Study of 25 Cases and Review of the Literature Findings. Turk Klin J Dermatol. 2013;23:77-83.

Copyright by Pinar Incel Uysal, et al. This is an open-access article distributed under the terms of the Creative Commons Attribution License, which permits unrestricted use, distribution, and reproduction in any medium, provided the original author and source are credited.

Source of Support: Nil, Conflict of Interest: None declared. 